\title{
Gaining Computational Literacy by Creating Hybrid Aesthetic Learning Spaces
}

\author{
Daniela Reimann \\ FORUM for Interdisciplinary Studies at the \\ Muthesius-Academy of Arts, Germany \\ e-mail:daniela@muthesius.de
}

\author{
Michael Herczeg, Thomas Winkler \\ Institute for Multimedia and Interactive Sys- \\ tems at the University of Lübeck, Germany \\ e-mail: herczeg@imis.uni-luebeck.de \\ e-mail: winkler@imis.uni-luebeck.de
}

\author{
Ingrid Höpel \\ Institute of Art History, University of Kiel, Germany \\ e-mail: ihoepel@kunstgeschichte.uni-kiel.de
}

\begin{abstract}
Although the technical skills of pupils are quite high, the current approach to gain media literacy still focuses on updating software applying skills, rather than exploring the potential for learning and the nature of digital media as programmed entities. Most educational research was focussed on the computer simply as a tool or a re-

\section{Introduction}

"By computational literacy I do not mean a familiarity with a machine that computes." [2]

One of our main goals is the development of models to establish a broader concept of computational literacy including the human as a holistic being by addressing the diverse senses of perception. Most educational research was focused on the computer simply as tool or a resource for archiving and accessing data. On the basis of our research undertaken in general education, two models of scenarios are introduced ${ }^{1}$, which are based on the idea of understanding the nature of digital media as programmed entities by shaping hybrid aesthetic learning spaces selfactingly.
\end{abstract}

\section{Shaping aesthetic Mixed-Reality learning spaces}

The concept of Mixed Reality allows for addressing the different human senses.

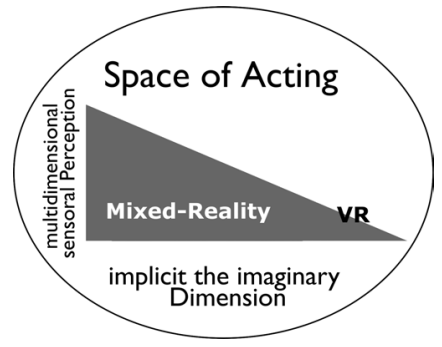

Fig. 1 The concept of Tangible Media, that is, the interface getting embedded in the objects of the physical world. The latter is perceived as an augmentation of the physical world. source, rather than on the idea of making transparent informatic modeling. It discusses the impact of selfmade hybrid learning environments to establish a multidimensional media literacy involving different human senses, aesthetic objects and artefacts.

Hybrid media art projects are often coined by a multidimensional and interdisciplinary approach to arts, crossing the borders between the disciplines such as arts, design, engineering, science, information and communication technologies, philosophy, literature as well as dramatic narratives and performance. Although the open concept and the variety of different work methods is opening up important impulses for learning, educational research seems to have missed scrutinizing the concept of Mixed-Reality for education purposes.

\section{Creating interactive aesthetic environments with Tangible Media}

Hard- and software tools are available on the consumer market, such as image and gesture recognition software. Only little research has been undertaken in exploring micro computers, robots, robotic toys and sensor technologies for learning such as the works by Druin and Hendler [3], or the works with tangible interfaces by Ishii, Patten and Griffith [4], who introduced the research field of Tangible Media at MIT.

As touching things is a means towards investigating and understanding the world, pupils are encouraged to learn about media by means of graspable objects, rather than reducing their activity to mouse click and key board activities. Tangible media support the interface to get activated in the very situation where learning takes place. A more natural way of interacting with the computer, such as those activated by using hand gestures, motion and body stance of any kind. By making transparent the invisible inside of computer in order to facilitate an overall understanding of algorithms and computational modelling. The work processes are coined by collaboratively developing concepts and aesthetic objects which are

\footnotetext{
${ }^{1}$ See ArtDeCom Model-Project on: http://artdecom.mesh.de
} 
brought together in the overall hybrid learning space. Pupils learn to instruct micro computers and test the programmes developed in the physical world. A variety of sensors such as those responding to temperature, haptic click or light have been given to the pupils to experiment with.
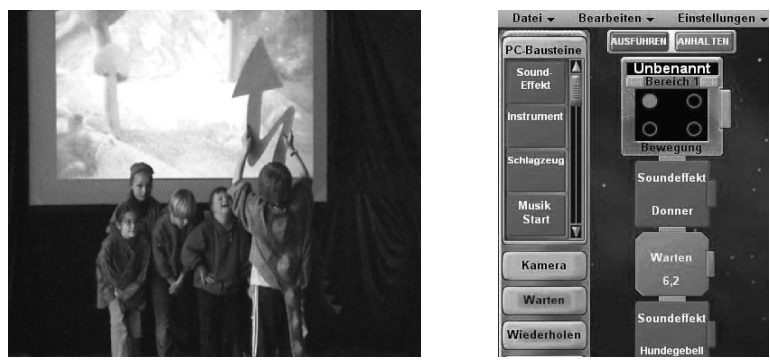

Fig. 2 Interactive hybrid learning spaces realised with sensor technologies and iconic programming on primary school level as well as on academic secondary level

\section{Experiencing Internet-worlds as avatars}

The development software for three-dimensional worlds delivered through the Internet has become affordable for school kids. Moreover, the pupils are familiar and highly skilled using such development tools. The main opportunity offered by virtual spaces is in terms of experiences which differ from such made in the physical environment. The software comes along with a construction tool mediating different approaches to threedimensional imagination of the pupils, such as player-, top-, side- and isometric view of construction level. The self-created world can be tested and experienced collaboratively using the software's player (viewer) modus. After the construction phase the collaborative navigation of the three-dimensional Internet-world representing the opening of an online-event, such as an exhibition. The latter can be put on stage in the context of an online event such as an the opening of an exhibition. Data projection allows for linking back virtual space to the physical environment as shown in figure 3.

The introduction of avatars add a new dimension to online communication. Avatars are digital representations of one's identity in synchronous communication. They can be created self-actingly. Furthermore, they can exist as independent entities as part of a virtual environment. In the latter they are representing a range of programmed motion and interaction looped in a particular context. A wide range of hand gestures and body movements linked to human conversations coined by expressions of anger, joy, flirt and the like can be simulated. Different forms of embodiments exist, such as two-dimensional, static ones as well as dynamic avatars which are able to move and communicate by using gestures, voice and the like.
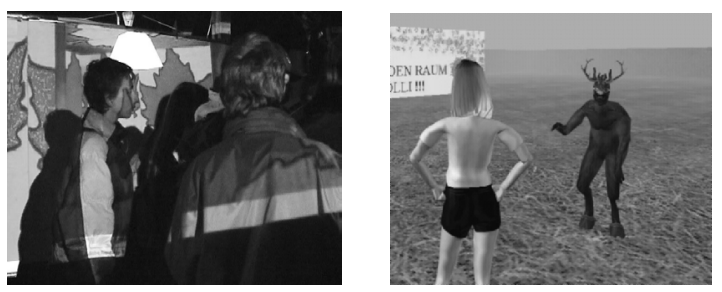

Fig.3 Projection of an Internet world as a part of a hybrid aesthetic learning space developed by $8^{\text {th }}$ graders of a comprehensive school (left); Pupils of 8th grade communicating online as avatars by using hand gestures and body stance

\section{Conclusion}

As the evaluation has shown Mixed-Reality learning spaces were proved being fruitful at the different age levels applied. The interviewees highlighted the importance of project working in the context of multimedia and the arts context. The evaluation has proved right the combination of algorithmic machine in the context of aesthetics and design being an excellent means to get the pupils involved in the learning content. On the long run, the updating of technical skills can not be perceived as the main educational goal. We believe in the necessity of a broader concept of media literacy which would be embedded in terms of constantly accompanying the students through their lifelong education careers. The computer needs to get embedded into new forms of project oriented learning, rather than simply being a tool for data processing and applying software. A new culture of learning has to be established on the long run. The combination of algorithmic machine and aesthetic learning spaces consists of a variety of stimulations in terms of aesthetics, social processes and interaction between the learners. The hybrid learning space is located in the very situation where learning is considered best to take place, be it a studio or the like. Hybrid aesthetic learning spaces allow for crossing the barriers of curricula by facilitating subject-crossing and project-oriented learning.

\section{References}

[1] Altstatt, R. (ed.): (2001), Avatars and others - Dan Graham, Lynn Hershman, Markus Huemer, Kristin Lucas, Victoria Vesna, Edith Russ Site for Media Art, Oldenburg, Frankfurt am Main: Revolver-Archiv für aktuelle Kunst

[2] DiSessa, Andrea A.: Changing minds - Computers, learning, and literacy, Massachusetts Institute of Technology, 2000 (MIT Press)

[3] Druin, Allison, Hendler, James: (2000) Robots for kids exploring New technologies for learning, San Diego, CA,

[4] Patten, James; Griffith, Laurie; Ishii, Hiroshi: A tangible Interface for controlling robotic toys, paper presented at the CHI'00, April 1-6, 2000, The Hague, The Netherlands 